\title{
New Exotic Models of Cold Nuclear Reactions and the Creation of the World
}

\author{
Andrey B. Simakov \\ National Research Nuclear University (NRNU MEPhI), Moscow, Russia \\ Email: absimakov@mephi.ru
}

How to cite this paper: Simakov, A.B. (2018) New Exotic Models of Cold Nuclear Reactions and the Creation of the World. Journal of High Energy Physics, Gravitation and Cosmology, 4, 504-509. https://doi.org/10.4236/jhepgc.2018.43028

Received: May 5, 2018

Accepted: July 3, 2018

Published: July 6, 2018

Copyright $\odot 2018$ by author and Scientific Research Publishing Inc. This work is licensed under the Creative Commons Attribution International License (CC BY 4.0).

http://creativecommons.org/licenses/by/4.0/

(c) (i) Open Access

\begin{abstract}
The work has the characters of a philosophical note, in which a new exotic version of the atom structure is discussed. According to this, the atomic nucleus consists of "normal" and "special" neutrons. Electrons are internal part of both types of neutron. Electrons can leave "normal" neutrons of the nucleus and return back with a certain probability. These processes lead to the appearance of protons in the nucleus and form the electron orbits of the atom. At the same time, it is possible that the Coulomb's barriers of atoms and nucleus disappear at some point in time and cold nuclear reactions pass through. This assumption leads to a new exotic model of the Universe structure, namely, the existence of neutron ether, consisting of special neutrons that do not emit their own electrons. In this ethereal ocean of special neutrons, periodically provoked disturbances arise. After that, it creates pockets, clusters of our normal neutrons inherent in our world, which can already emit electrons and, consequently, create atoms. The ether gets sick from time to time. However, as a result of this disease, stars arise. Some possible stages in the creation of our world are also discussed in this paper.
\end{abstract}

\section{Keywords}

Low Energy Nuclear Reaction, Coulomb Barrier, Neutron, Proton, Electron Vacancy, Neutron Ether, Dark Matter

\section{Introduction}

At the beginning of this note, it should be reported that its author has an indirect relation to the problems of theoretical nuclear physics, although he works at the NRNU MEPhI and performs some works on applied nuclear research [1] [2] and experimental high energy physics [3] [4]. Also, the first question of this report is the problem of low-energy nuclear reactions (LENR) or the catchier 
name "cold nuclear fusion", which is one of the most controversial scientific fields of advanced nuclear physics. On the one hand, there is a growing number of theoretical and experimental publications from world-renowned research centers and laboratories and official announcements of LENR as a pseudo-science from some official commissions. However, it should be noted that in the recent past, certain official commissions have also declared false scholars by Galileo and, especially, Giordano Bruno. As an author from Russia, I would like to remember that more than 100 years ago, Vladimir Lenin, the great propagandist of the dialectical approached to everything, including nuclear physics, said the winged phrase: "Electron is as inexhaustible as the atom ......". Therefore, respecting the opinion of Vladimir Lenin, I believe that working on phenomena that do not have a classical explanation at the moment, the case is ungrateful, but admissible.

Additionally, the inexplicability of LENR is primarily connected to the axiom about the impossibility of overcoming the Coulomb barrier and subsequent nuclear interactions in the classical model of the atom structure. This verdict LENR also rendered more than a hundred years ago the father of nuclear physics Ernest Rutherford. In attempts to appeal this sentence, dozens of theoretical models were proposed. In the recent issue of the Russian journal RENSIT [5], several articles with a classical LENR approach were collected, but with seditious conclusions about the possibility of "impossible". In one of the survey works, it was even noted that all sorts of exotic (not classical) models and theories (although there are many of them) are not accepted for consideration as pseudoscientific. According to this, among the theoretical marginal, there are even more irregular marginal. Nevertheless, I, climbing into a group of these extreme exotic assumptions, want to propose a new non-quantitative and qualitative exotic model of cold nuclear reactions, which can and should be "chopped into cabbage”, but at the same time dialectically take into account.

\section{Some Exotic Changes in the Classical Model of the Atom}

The new model does not break the basic postulates of the planetary atomic model, but the following "frightening" assumptions are advanced:

1) nucleus of an atom consists only of neutrons; the protons are neutrons with a "hole", i.e. with the missing electron ("hole"-the term of physics of semiconductors);

2) positive charge is a vacancy corresponding to a virtual particle that does not exist without an electron;

3) electrons leave the some "normal" neutrons of the nucleus with a certain probability and return to the nucleus also with some probability; these are fast atomic processes (note, that a free neutron decays with a half-life of about 11 minutes);

4) principle of Pauli allows electrons to pair in separate energy atomic orbits (according to the classics); 
5) the probability of finding an electron either in the nucleus or in the orbit leads to the appearance of the probability of a complete absence of electrons in the orbits of the atom, i.e. the absence at any time of the orbital Coulomb protection of the atom; moreover, the situation "all electrons in the nucleus" means the absence of a charge of the nucleus itself, and therefore the absence of a nuclear Coulomb barrier, and this is the condition for the passage of the LENR;

6) processes of combining nuclei into so-called "trans-atoms" and "trans-molecules" and their subsequent transformations can occur according to the principles described in [5].

Of course, the proposed exotic model has only a qualitative character and requires its physical quantitative and experimental confirmation. However, she explains why "Bohr" electrons do not "fall" on the nucleus under the influence of the same electrostatics. They always fall, but they always return back to the "Bohr's orbits". What conditions under? That is the question. By the way, one of the most respected classical works [6] says: "We think that a neutron, at least at some points in time, looks like a proton surrounded by a cloud of negative $\pi$-meson." No less exotic conclusion of the proposed model would be a logical negation of such a particle as a positron and its consideration as an electron vacancy in space, i.e. return to the old, long-forgotten and rejected theory of spatial ether.

\section{Exotic Model of Spatial Neutron's Ether and Possible Stages of World Creation}

The life truth says: "Nature does not tolerate emptiness." However, practically the whole world, the entire universe is only emptiness-vacuum (radiation does not yet considered). It is said the same in classical physics. However, if the atom partly consists of "normal" neutrons, throwing electrons into atomic orbits, then we can assume that everything around is filled with "special" neutrons that never emit orbital electrons. Therefore, they fill the ether, interacting with each other under other exchange laws. Also, we, our world, live in an ocean (space) of special neutrons. Classics will say- "crazy house". Here they are-neutron stars, gravitational monsters because of the huge mass, not even emitting light. However, what if they do not produce it at all? Also, the physical mass appears only in the interaction, but it simply does not exist in the special neutrons of the ether and matter of our world. Moreover, the mass is a characteristic not known for such ethereal neutrons. It is possessed only by normal neutrons of our material world. Remember-the matter is given to us in sensations, or it is possible to continue-in the indication of our created instruments. If is not given in sensations, then what? Then the ether, that dark matter, in search of which not a single generation of classical physicists has turned gray, and which has not been given to us yet in either sensations or indications of instruments. What led to the transition of special neutrons to normal ones and why don't they return? That is the question. Not returning? They are returning, with the cooling of the Un- 
iverse our matter is gradually disappearing into the "black holes" of the ether. Then, how about our native, our felt matter-our world? When will it end? The classic says- "Big Bang"- and she does not believe it. If you stand on the position of the special neutron's ether, then in this calm ethereal ocean, there are occasional disturbances, something provoked. Then, it effervesces, creating clusters, clots of normal neutrons, possibly mixed with special ones. The ether gets sick from time to time. However, as a result of this disease, stars arise. This ether disease can take place on a cosmic scale, for example, a thousand or even a million years. Then, on the same scale, it's an explosion (Big Bang). How can I not remember the Bible-and God created the light, and God created the Earth. Bunches of normal neutrons, suddenly began to have the characteristics of our matter habitual for us, and it started. Nuclear reactions began; the first atoms began to be created. A number of star clusters received good rotational motion at birth, and the centrifugal forces that emerged in the new world began to pull out of them pieces that already were distributed around the stars according to Kepler's laws. It should be noted that young gaseous planets scattered their pieces, creating personal satellites, for example, the Moon. One of the young planets could not withstand the gravity that arose together with the world, scattered into parts, so the asteroid belt of the solar system arose later. The planets began to cool down, radiating into space, then light and other radiation. However, they cooled down differently, creating themselves as a result of high-temperature thermonuclear fusion, everything that forms today Mendeleev's table. On planets distant from the sun, this cooling process took place more quickly. They have remained in the form of gas giants or ice blocks, as well as another type of cold star wreckage-comets. When the planets cooled down, the thermonuclear synthesis of atoms gave way to inorganic chemistry. The earth has become a physicochemical reactor. Complex chemical compounds, stable under specific temporary physical and chemical conditions, began to appear. Then the time of organic chemistry came, with its molecular complexes of the most common elements-carbon and hydrogen. It should be noted that the problem of the appearance of hydrocarbons, our present energy wealth, as well as LENR, has split modern science. D. Mendeleev, mentioned above, became a consistent supporter of the abiogenic hypothesis, i.e. continuous creation of hydrocarbons, in particular, oil in deep bowels of the Earth [7]. This is in contradiction with the dominant world theory of the origin of oil-a biogenic theory, according to which it was formed from the remains of ancient living organisms. [8]. However, if in the geological physical and chemical evolution of the Earth conditions (temperature, pressure, lighting, external radiation, catalysts, etc.) have arisen in which (their duration is estimated at 50 - 300 million years) organic chemistry has become a natural tool for creating "useful" hydrocarbons in the surface layers and on the surface of the planet, then the abiogenic theory acquires a new meaning. Moreover, the emergence of complex organic molecules under the conditions of the appearance of "liquid" water led to the formation of the first organic cells, 
which, using external energy, became self-reproducing, i.e. there was a life. No wonder the majority of living organisms consists of water and dies at the temperature of its boiling, and boiling water is successfully used for sterilization in medicine. Therefore, the appearance of the earth's oceans became a condition for the emergence of life from the previously prepared organic environment. Also, the numerous offensive and retreat of the water oceans on the sea of oil created and divided oil deposits over the surface and density. Therefore, light oil, as a rule, lies closer to the earth's surface, and the heavier one is deeper. Therefore, probably, first there were the seas of oil and organic, and then the oceans of water and the living. Returning to the processes of ether's perturbations, these processes of creating new stars and new galaxies according to dialectics should continue, and, God forbid, that it would happen again next to us. The stars die and are born again continuously. And in the specific, "dead" neutrons of the ether is a terrible energy. Her awakening is equivalent to the birth of a star, which is observed by astronomers as a supernova flash. Maybe that's why modern experiments on the study of matter in extreme states on large collider installations (BNL, CERN, FAIR) cause vague apprehensions of many physicists. Will they, even in small space, cause the reaction of the transformation of the specific, sleeping neutrons of the ether into ours-normal, ready immediately, without cooling, to begin creating a new world. Little does not seem.

\section{Conclusions}

The suggested assumption that the electron orbits of atoms are created by the probabilistic process of electron emission by the neutrons of the nucleus leads to the possibility of the absence at some times of the Coulomb barriers of the atom, the critical approach of the nucleus and the passage of cold nuclear reactions. A look at a proton as a neutron with an electron vacancy (a "hole") leads to the hypothesis of the existence of a neutron ether (dark matter). The "special" neutrons make it do not emit the orbital electrons and therefore do not participate in the physical interactions that are customary for our world. Periodic perturbations of the dark ether lead to the creation of "normal" neutrons clusters of our world, which emit electrons, create atoms and nuclear reactions in the composition of stars and other cosmic objects. The evolution of the planets of the solar system was determined by the dominance of physical, chemical and geological conditions, and if nuclear reactions predominated at the initial stage, then the era of inorganic and organic chemistry came. Linking cosmology to the modern problem of LENR, I would like to note that these are already pathetic remnants of those old processes that "create planets iron" while it was hot. And, by the way, the observed neutron fluxes in high-power lightning discharges can be related to the high-voltage perturbation of the neutron ether.

Additionally, how can one not remember the genius Nikola Tesla, who with his high-voltage experiments looked into the abyss of the ether, was horrified by the possible consequences of his awakening, and ...... and fell silent. 
This publication is provocative and built on exotic assumptions; but it is primarily aimed at continuing discussions on the controversial issues of the existence of both LENR and the space ether.

\section{References}

[1] Gurkovskiy, B.V., Onishchenko, E.M. and Simakov, A.B. (2015) Long-Distance Detection of Alpha-Radioactivity: Method and Device. Journal of Environmental Radioactivity, 149, 150-157. https://doi.org/10.1016/j.jenvrad.2015.07.019

[2] Bogdanovich, B. and Simakov, A. (2015) Oxygen Isotopes Application for Growing of Silicon Dioxide Films with Raised Radiation Hardness. Proceedings of the Conference on Advances in Radioactive Isotope Science (ARIS2014), 6, Article ID: 030145. https://doi.org/10.7566/JPSCP.6.030145

[3] Voronov, S.A., Voronov, Yu.A., Onishchenko, E.M., Simakov, A.B. and Sugrobova, T.A. (2004) High-Voltage Safety Fuses for the Transition-Radiation Tracking Detector in the ATLAS Experiment. Instruments and Experimental Techniques, 47, 191-193. https://doi.org/10.1023/B:INET.0000025200.74310.60

[4] Atkin, E., Bocharov, Yu., Butuzov, V., Klyuev, A., Osipov, D., Shumikhin, V., Simakov, A. and Voronin, A. (2010) Development of a Data-Driven Readout ASIC for Microstrip Detectors. 2010 East-West Design \& Test Symposium (EWDTS), St. Petersburg, 17-20 September 2010, 23. https://doi.org/10.1109/EWDTS.2010.5742121

[5] RENSIT: Radio-Electronics. Nano-Systems. Information Technologies, 2017, 9, № 1. http://rensit.ru/vypuski/article/200/9(1)1-128.pdf

[6] Feyman, R., Leighton, R. and Sands, M. (1966) Feynman's Lectures on Physics. Electrodynamics. Publishing House "MIR", Moscow, 320.

[7] Sephton, M.A. and Robert, M.H. (2013) On the Origins of Deep Hydrocarbons. Reviews in Mineralogy and Geochemistry, 75, 449-465. https://doi.org/10.2138/rmg.2013.75.14

[8] Höök, M., Bardi, U., Feng, L. and Pang, X. (2004) Development of Oil Formation Theories and Their Importance for the Sake of Oil. Marine and Petroleum. Geology, 27, 1995-2004. 